INPLASY

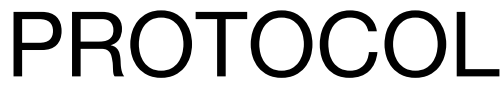

To cite: Zhang et al. Shenling Baizhu powder improves intestinal microecological environment in different patients: a protocol for systematic review. Inplasy protocol 202160089. doi: 10.37766/inplasy2021.6.0089

Received: 23 June 2021

Published: 23 June 2021

Corresponding author: Xiaofan Chen

xiaofanci122306@163.com

Author Affiliation: Jiangxi University of Chinese Medicine.

Support: Grant No. 20192BAB205095.

Review Stage at time of this submission: Formal screening of search results.

Conflicts of interest:

None declared.

\section{Shenling Baizhu powder improves intestinal microecological environment in different patients: a protocol for systematic review}

Zhang, R1; He, Q2; Yi, X3; Chen, S4; Chen, Q5; Yu, Q6; Zhang, J7; Dong, J8; Zhang, $\mathrm{H}^{9}$; Chen, $\mathrm{X}^{10}$.

Review question / Objective: Shenling Baizhu powder improves intestinal microecological environment in different patients.

Information sources: We searched PubMed database, web of Science (SCI), Cochrane Library, CNKI database, VIP database and Wanfang database. The key words were "Shenling Baizhu powder", "intestinal flora" in Chinese and "Shenling Baizhu powder", "slbzs" and "gastroenteric microorganism" in English. The retrieval time was set from the date of database establishment to April 15, 2021. No language or publication restrictions were set, and search terms were modified in different databases to identify all possible matching clinical trials. PubMed's search strategy is shown in Table Table1.1. These search terms will be accurately translated into other databases.

INPLASY registration number: This protocol was registered with the International Platform of Registered Systematic Review and Meta-Analysis Protocols (INPLASY) on 23 June 2021 and was last updated on 23 June 2021 (registration number INPLASY202160089).

\section{INTRODUCTION}

Review question / Objective: Shenling Baizhu powder improves intestinal microecological environment in different patients
Rationale: PubMed, web of Science (SCI), Cochrane Library, CNKI, Wanfang database, VIP database and CBM database were searched to collect the clinical experimental research related to Shenlingbaizhu powder intervention on 
intestinal flora. The search time was from the establishment of the database to May 30,2021 . No language or publication restrictions. Two reviewers independently screened the literature and extracted the data, and used Cochrane assessment manual 5.4.1 to evaluate the risk of the literature in line with this study. We will use Stata v 16.0 software to conduct metaanalysis.

Condition being studied: Shenling Baizhu Powder(SLBZS) is a canonical spleentonifying Chinese herbal formula used to treat chronic diarrhea and poor appetite, which was first documented in "Tai Ping Hui Min He Ji Ju Fang" in Song-dynasty. The collection of bacteria, archaea and eukarya colonising the GI tract is termed the 'gut microbiota' and has co-evolved with the host over thousands of years to form an intricate and mutually beneficial relationship. The number of microorganisms inhabiting the GI tract has been estimated to exceed 1014, which encompasses $\sim 10$ times more bacterial cells than the number of human cells and over 100 times the amount of genomic content (microbiome) as the human genome. Some studies have shown that Shenling Baizhu powder has certain effect in improving animal intestinal flora. Shaobao Zhang et al. Found that slbzs treatment reversed the intestinal imbalance of FD. With SLBZS treatment, FD biomarkers including Prevotella, Mucispirillum and Akkermansia were decreased while SCFA-producing bacteria such as Adlercreutzia and Clostridium, and sulfate-reducing bacteria Desulfovibrio were enriched. Additionally, SLBZS normalized the dysregulated function of the microbiome, upregulating the pathways of energy metabolism and decreasing the oxidative stress as well as bacterial pathogenesis. Based on the results of 165 rRNA, Wei Jie LV et al. Found that slbzs treatment could change the structure of intestinal microflora, thus enhancing the richness and diversity of intestinal microflora in DSS model rats. SLBZS seems to offset the structural changes brought about by DSS. However, the intervention effect of Shenling Baizhu powder on human intestinal flora is relatively less, and most of the study population is the same type of patients. However, the intervention effect of Shenling Baizhu powder on human intestinal flora in different patients is worth exploring, and there is no evidence-based medical research support at present. Therefore, it is necessary to systematically evaluate the intervention effect of Shenlingbaizhu powder on human intestinal microecological environment in different patients.

\section{METHODS}

Search strategy: PubMed, web of Science (SCI), Cochrane Library, CNKI, Wanfang database, VIP database and CBM database were searched to collect the clinical experimental research related to Shenlingbaizhu powder intervention on intestinal flora. The search time was from the establishment of the database to April 2021. No language or publication restrictions. Search strategy used in PubMed database. \#1:"Gastrointestinal Microbiome" [mesh]; \#2: (Gastrointestinal Microbiomes) OR (Microbiome, Gastrointestinal) OR ( Gut Microbiome) OR ( Gut Microbiomes) OR ( Microbiome, Gut) OR ( Gut Microflora) OR ( Microflora, Gut) OR ( Gut Microbiota) OR ( Gut Microbiotas) OR ( Microbiota, Gut) OR (GastrointestinalFIora) OR (Flora, Gastrointestinal) OR (GutFlora) OR (FIoraGut) OR (Gastrointestinal Microbiota)OR (Gastrointestinal $M$ i c robiotas) OR ( $M$ i crobiota, Gastrointestinal) OR(Gastrointestinal Microbial Community) OR(Gastrointestinal Microbial Communities)OR(Microbial Community, Gastrointestinal)OR (Gastrointestinal Microflora) OR (Microflora, Gastrointestinal) OR (Gastric Microbiome) OR (Gastric Microbiomes) OR (Microbiome, Gastric) OR (Intestinal Microbiome) OR (Intestinal Microbiomes) OR (Microbiome, Intestinal) OR (Intestinal Microbiota) OR (Intestinal Microbiotas) OR (Microbiota, Intestinal) OR (Intestinal Microflora) OR ( Microflora, Intestinal) OR ( Intestinal Flora) OR ( Flora, Intestinal) OR ( 
Enteric Bacteria) OR ( Bacteria, Enteric); \#3: "shenling baizhu powder" [mesh]; \#4: (shenling baizhu powder) OR (slbzs) OR (Can Zuo Bai Shu San); \#5: \#1 OR \#2; \#6: \#3 OR \#4; \#7: \#5 AND \#6.

Participant or population: Adults taking Shenlingbaizhu powder.

Intervention: The study group was divided into control group and observation group. The observation group was intervened by other products such as Shen Ling Baizhu powder or Shen Ling Atractylodes rhizome.

Comparator: The control group was given other intervention. The dose, route of administration or follow-up time of intervention were not limited.

Study designs to be included: 1.Type of studies. This review will include randomized controlled trials (RCTs), whether using blinding.2.Type of participants.Adults taking Shenlingbaizhu powder.3.Types of interventions. The treatment group was treated with Shenling Baizhu powder (decoction, granule, pill, tablet, injection).4.Type of comparators. The control group was given other intervention. The dose, route of administration or follow-up time of intervention were not limited.5.Types of outcome measures. The main outcome measures included the number of Lactobacillus, Bifidobacterium, Escherichia coli and EnterococcusEnteroc

Eligibility criteria: Studies that meet one of the following criteria will be excluded: 1)Republished literature. 2) Animal experimental study 3)Research data is incomplete or full text is not available. 4)Study on the type of participants not meeting the requirements.

Information sources: We searched PubMed database, web of Science (SCI), Cochrane Library, CNKI database, VIP database and Wanfang database. The key words were "Shenling Baizhu powder", "intestinal flora" in Chinese and "Shenling Baizhu powder", "slbzs" and "gastroenteric microorganism" in English. The retrieval time was set from the date of database establishment to April 15,2021 . No language or publication restrictions were set, and search terms were modified in different databases to identify all possible matching clinical trials. PubMed's search strategy is shown in Table Table1.1. These search terms will be accurately translated into other databases.

Main outcome(s): The main purpose of Shenlingbaizhu powder intervention is to improve the micro ecological environment of intestinal flora. Therefore, the change of intestinal flora composition is the main observation index, including the colony number of Lactobacillus, Bifidobacterium, Escherichia coli and Enterococcus.

Additional outcome(s): Secondary outcome measures included intestinal barrier function, such as short chain fatty acids, Dao, D-lactic acid, etc.

Data management: EndnoteX9.1 software will be used by us to exclude duplicate studies. Two independent researchers will screen the literature after removing duplicates. Inconsistent opinions will be resolved through discussions with the third researcher. The selection process will be shown through the PRISMA chart. Two researchers independently extracted data based on predesigned forms and crosschecked them. The extracted content includes the first author, title, publication time, age, disease type, sample size, intervention measures, outcome indicators, and related data.

Quality assessment / Risk of bias analysis: Methodological quality for each included trial will be assessed using the tools of Cochrane Handbook for Systematic Reviews of Interventions. The bias risk assessment category will include the following 7 areas: randomized sequence generation; allocation concealment; blinding of participants; blinding of outcome assessors; incomplete outcome data; selective outcome reporting; other bias. Two independent risk assessments of bias were conducted on the literature, and any differences would be discussed and resolved with the third researcher. Each 
assessment is labeled "high risk", "low risk" or "unclear risk".

Strategy of data synthesis: We will use Stata v 16.0 software to conduct metaanalysis. If it is continuous data, it will be calculated based on the mean difference (MD) of the $95 \%$ confidence interval (CI), and the dichotomous data will be calculated based on the risk ratio (RR) of the $95 \% \mathrm{Cl}$. Chi-Squared test and 12 test were used to test the heterogeneity of the included literature. When $P>0.1$ and $12<$ $50 \%$, it indicates that there is no statistical heterogeneity between the studies; conversely, when $\mathbf{P}<0.1$ and $12>50 \%$, it is considered that there is statistics heterogeneity between the studies. Less than 10 studies will not be analyzed for reporting bias. If more than 10 studies are included, the symmetry of the funnel chart will be used to detect potential reporting bias.

Subgroup analysis: If possible, we will include the study for grouping analysis.

Sensitivity analysis: In order to evaluate the robustness of data analysis, sensitivity analysis will be performed.

Language: No language or publication restrictions.

\section{Country(ies) involved: China.}

Keywords: protocol; Intestinal Microecology; Shenling Baizhu Powder; systematic review.

Contributions of each author:

Author 1 - Ruirong Zhang.

Email: zhangrr0388@163.com

Author 2 - Qingying He.

Email: hqy2019free@163.com

Author 3 - Xingqian Yi.

Email: xiaoyi19861030@163.com

Author 4 - Shuxian Chen.

Email: zzj199593@163.com

Author 5 - Qian Chen.

Email: cc77chen@126.com

Author 6 - Qinqin Yu.

Email: 2366414921@qq.com
Author 7 - Jiahui Zhang.

Email: 81095119@qq.com

Author 8 - Jiawei Dong.

Email: 921678802@qq.com

Author 9 - Hua Zhang - Corresponding author.

Email: 20191002@jxutcm.edu.cn

Author 10 - Xiaofan Chen - Corresponding author.

Email: xiaofanci122306@163.com 